\title{
Comparison of traditional 2D and 3D Omniview technique in detection of fetus corpus callosum
}

\author{
Yang Li ${ }^{1}$, Guo-wei Tao' ${ }^{2}$ Ming-zhi Pan ${ }^{3}$, Guang-rui Shao ${ }^{4}$
}

${ }^{1}$ Obstetrics and Gynecology Hospital of Provincial Hospital Affiliated to Shandong University, Key Laboratory of Birth Regulation and Control Technology of National Health and Family Planning Commission of China, Key Laboratory for Improving Birth Outcome Technique, Shandong Provincial Family Planning Institute of Science and Technology, ${ }^{2}$ Qilu Hospital of Shandong University, ${ }^{3}$ Shandong Maternal and Child Health Hospital, ${ }^{4}$ The Second Hospital of Shandong University, Jinan, Shandong, China

\begin{abstract}
Aim: To explore the value of Omniview (OV) technology in assessing the fetus corpus callosum. Material and methods: For the ultrasound examination of 189 fetuses (gestational week range 19-28 weeks), two-dimensional (2D) ultrasound imaging and Omniview technology were used by two physicians (A and B). The acquisition time and the quality of images were recorded. Results: The acquisition time of Omniview technology was shorter comparing with the aquisition time of 2D ultrasound imaging $\left(\mathrm{A}_{2 \mathrm{D}} v s . \mathrm{A}_{\mathrm{OV}}: 159.44 \pm 27.09 \mathrm{~s} v s .73 .90 \pm 18.99 \mathrm{~s}, \mathrm{p}<0.01 ; \mathrm{B}_{2 \mathrm{D}} v s . \mathrm{B}_{\mathrm{OV}}: 120.22 \pm 21.89 \mathrm{~s} v s .74 .19 \pm 14.86 \mathrm{sec}-\right.$ onds, $\mathrm{p}<0.01)$. 2D ultrasound examination was performed in a longer time by the junior physician, compared with the senior physician $\left(\mathrm{A}_{2 \mathrm{D}}\right.$ vs. $\mathrm{B}_{2 \mathrm{D}}: 159.44 \pm 27.09 \mathrm{~s}$ vs. $\left.120.22 \pm 21.89 \mathrm{~s}, \mathrm{p}<0.01\right)$. The intra- and inter-observer reliability of acquisition time using Omniview technology was good (LoA: -11.8 to $+12.4 \mathrm{~s}$, and -41.9 to $+43.3 \mathrm{~s}$, respectively). The success rate of junior and senior physicians with the Omniview technique was $76.2 \%$ and $80.4 \%$, respectively. There was good consistency between the Omniview technique and 2D ultrasound imaging in terms of image quality (Kappa $=0.782,95 \%$ CI: 0.586-0.977). Conclusion: Omniview technique can not only obtain corpus callosum images more effectively but also can achieve better quality images. Therefore, it is a reliable method to image the fetal corpus callosum.
\end{abstract}

Keywords: Omniview; fetus; ultrasonography; corpus callosum

\section{Introduction}

The complexity of the brain lies in its connection, this is highlighted by the disproportionate increase in white matter volume throughout primate evolution [1]. The largest connective structure in the brain is the corpus callosum; it consists of over 200 million fibers connecting the two brain hemispheres [2]. The corpus callosum contains homotopic and heterotopic interhemispheric connections [3]. Studies have shown that the difference

Received 26.04.2018 Accepted 12.09.2018

Med Ultrason

2018, Vol. 20, No 4, 493-497

Corresponding author: Guang-rui Shao

Department of Radiology

The Second Hospital of Shandong University

Jinan, China

E-mail: 77363741@qq.com between the size and shape of the corpus callosum is correlated with the difference in the cognitive ability of the brain [4]. Therefore, determining the normal development of the corpus callosum is important during the examination of the fetal central nervous system.

Fetal MRI can assess the development of the fetal nervous system, but it is expensive, time consuming and is limited by fetal movement and maternal respiration [5]. Ultrasound has the advantages of safety, economy, real-time and reproducibility. It is the first choice for evaluating fetal brain development [6]. At present, the diagnosis of corpus callosum agenesis mainly relies on two-dimensional (2D) ultrasound imaging. However, the image quality is affected by many factors such as fetal position, gestational week, maternal abdominal thickness, physician's experience and amniotic fluid. Furthermore, the assessment is based on indirect symptoms such 


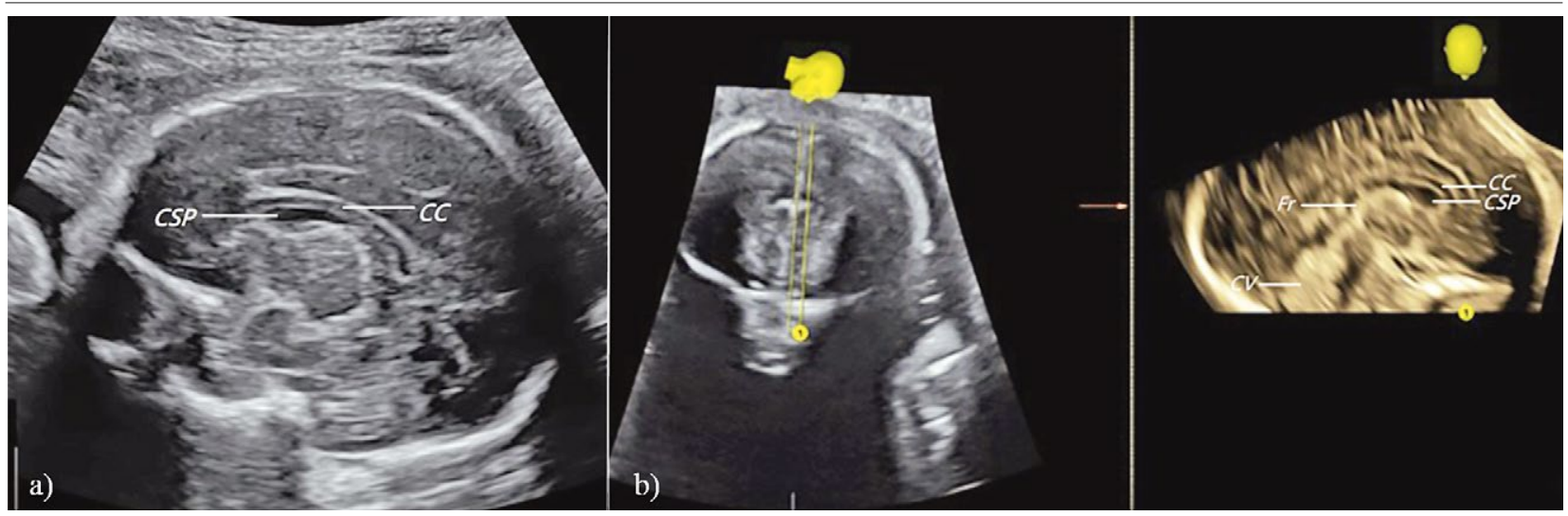

Fig 1. A 28 weeks, fetus: a) 2D ultrasound image of the fetus, which displays the genu, body and splenium part of the complete corpus callosum (CC); b) the same fetus, showing the genu, body and splenium part of the corpus callosum (CC), providing more normal sagittal plane information of the fetus, such as the cavum septi pellucidi (CSP), cerebellar vermis (CV) and fornix (Fr).

as the absence of the cavum septi pellucidi, changes in the lateral ventricle, the expansion of the third ventricle, and the echo between the lateral ventricle and falx. Overall, the assessment of the corpus callosum using 2D ultrasound imaging remains to be challenging.

The use of three-dimensional ultrasound (3DUS) in Obstetrics and Gynecology has been reported in many published papers $[7,8]$. In recent years, there have been a continuous development in volumetric ultrasound imaging [9-12]. Omniview is a software application for 3DUS that allows interrogation of volume datasets and simultaneous display of up to three independent (nonorthogonal) planes by manually drawing line, curve, polyline or trace from any direction or angle [13]. The first line (yellow), the second line (fuchsia), and the third line (turquoise) should be applied to the region of interest, achieving non-orthogonal planes simultaneously displayed on 3D volume multiplanar rendering [14]. This compensates the shortcomings of other volumetric imaging techniques, in which only one of the three orthogonal planes may be obtained. Furthermore, this allows for better imaging of irregular fetal brain structures.

In the present study, imaging efficiency, success rate and image quality of the corpus callosum were analyzed by comparing traditional $2 \mathrm{D}$ ultrasound imaging with Omniview technology.

\section{Material and methods}

\section{Subjects}

A total of 189 pregnant women who underwent prenatal screening in our hospital between December 2015 and October 2016 were enrolled in the present prospective study. The study was approved by local Ethics Committee and the subjects signed an informed consent prior enrollment. All participants were confirmed with a single pregnancy. The gestational week ranged within 1928 weeks and the age of these pregnant women ranged within 20-43 years old. None of the participants had any abnormal obstetrical history or familial inheritance and regular menstrual cycle was confirmed for all female participants. The gestational week was collectively determined by the last menstruation period, fetus biological parameters and the lateral diameter of the cerebellum. No visible morphological abnormality is observed through 2D ultrasound imaging. Ultrasound imaging was performed using the GE Voluson E10 Doppler. The 2D transducer used had a frequency range of 1.6-4.5 MHz, while the 3D transducer used had a frequency range of 3.5-5.0 MHz. Transabdominal imaging was performed to obtain the volumetric data of the corpus callosum and was consequently reconstructed using Omniview.

\section{Ultrasound imaging}

In transabdominal 2D ultrasound imaging the midsagittal view of the fetus head was realized in three steps: 1) obtaining the biparietal diameter view of the fetus head; 2) rotating the transducer by $90^{\circ}$ to obtain a midline profile; 3 ) slightly swinging the probe and using the acoustic window of the frontal suture and the anterior fontanel to show the corpus callosum. The 3D ultrasound volumetric data were obtained in the coronal plane of the fetus head, a plane in which the thalamus and transparent septum could be clearly observed. After obtaining the optimal image section, the subjects were instructed to hold their breath to prevent fetal movement and respiratory motion. The $3 \mathrm{D}$ volumetric imaging was triggered and the field of view of the image was adjusted, allowing the entire brain to be covered. After obtaining the 3D structural image of the brain, multiplanar reconstruction was performed by choosing the A planar, followed by the volume contrast 
imaging in the C-plane (VCI-C) function. Omniview was selected, and manual outlining was performed vertically on the brain midline, resulting in the sagittal view of the fetal head (fig 1). If fetal motion was detected during the data acquisition, the image was reacquired.

\section{Acquisition time}

A junior (physician A) and senior (physician B) ultrasound physician made consecutive ultrasound examinations of the pregnant women. Both physicians had prenatal screening qualifications and were able to identify the basic structures of the normal thalamus, cavum septi pellucidi and corpus callosum. They were instructed how to use the Omniview technology to access the corpus callosum. The time need to acquire the corpus callosum was recorded. Each physician performed the imaging of the corpus callosum using 2D ultrasound imaging and Omniview technology, respectively. We established a maximum time for $2 \mathrm{D}$ and $3 \mathrm{D}$ acquisition of five minutes. If the physician could not obtain a correct imagine of the corpus callosum in this time the acquisition was considered to be a failure. The 2DUS imaging acquisition time $\left(\mathrm{A}_{2 \mathrm{D}}\right.$ and $\left.\mathrm{B}_{2 \mathrm{D}}\right)$ and Omniview technology acquisition time $\left(\mathrm{A}_{\mathrm{OV}}\right.$ and $\left.\mathrm{B}_{\mathrm{OV}}\right)$ for both physicians $(\mathrm{A}$ and $\mathrm{B})$ were collected. To evaluate the intra-observer reliability of Omniview technology, physician A reexamined all fetus in the same day, a few hours later $\left(\mathrm{A}_{\mathrm{OV}}\right)$.

\section{Evaluation of the corpus callosum}

The images were scored using a 3-point scale, as shown below: Excellent (3 points) - the overall contour of the corpus callosum could be clearly observed, and a clear delineation from the transparent septum could be obtained. The three parts of corpus callosum, including the genu, trunk and splenium, could all be clearly identified; Average ( 2 points) - the overall contour of corpus callosum viewed to a good extent, but was not clear and the delineation from transparent splenium was slightly blurred. The visualization of the corpus callosum may be impacted; Poor (1 point) - the overall contour of the corpus callosum could be observed to some extent and the delineation from the transparent splenium was blurred. The visualization of the corpus callosum would be impacted.

\section{Statistical analysis}

Measurements were presented as mean and standard deviation. The time difference in performing the imaging between these two physicians was compared by t-test.

The intra- and inter-observer reliability was analyzed by Bland and Altman plotting. It was used to assess the quality of concordance, with the upper and lower limits of agreement defined as the mean difference plus and minus 1.96 times the standard deviation of the differences.

Kappa test was used to analyze the consistency of the image quality in these two modes of imaging.

\section{Results}

\section{Success rates of $2 D$ ultrasound imaging and Omniview technology}

In the 2D ultrasound imaging of 189 fetuses, the junior physician successfully obtained 48 cases of corpus callosum images (success rate $25.4 \%$ ), while the senior physician successfully obtained 73 cases of corpus callosum images (success rate 38.6\%).

In the imaging of 189 fetuses using Omniview technology, the junior physician successfully obtained 144 cases of corpus callosum images (success rate 76.2\%), while the senior physician was able to obtain 152 cases of corpus callosum images (success rate 80.4\%).

\section{Results of acquisition times}

The imaging acquisition time of Omniview technology was shorter, when compared with 2D ultrasound imaging and the acquisition time of the junior physician was longer only for 2D ultrasound examination, comparing with the senior physician (table I).

\section{The intra- and inter-observer reliability}

of acquisition time using Omniview technology

Regarding intra-observer agreement, fig 2a shows Bland-Altman plots indicating a good agreement; the mean difference was $0.3 \mathrm{~s}$, LoA: -11.8 to $+12.4 \mathrm{~s}$. For the

Table I. Acquisition times using 2D ultrasound imaging and Omniview technology

\begin{tabular}{lll}
\hline & Acquisition time (s) & p \\
\hline $\mathrm{A}_{2 \mathrm{D}}$ vs $\mathrm{A}_{\mathrm{OV}}$ & $159.44 \pm 27.09$ vs $73.90 \pm 18.99$ & $<0.01$ \\
$\mathrm{~B}_{2 \mathrm{D}}$ vs $\mathrm{B}_{2 \mathrm{D}}$ & $120.22 \pm 21.89$ vs $74.19 \pm 14.86$ & $<0.01$ \\
$\mathrm{~A}_{2 \mathrm{D}}$ vs $\mathrm{B}_{2 \mathrm{D}}$ & $159.44 \pm 27.09$ vs $120.22 \pm 21.89$ & $<0.01$ \\
\hline
\end{tabular}

Data are presented as mean \pm standard deviation. The $2 \mathrm{D}$ ultrasound imaging acquisition time (A2D and B2D) and Omniview technology acquisition time (AOV and BOV) for both physicians (A and B) are presented.

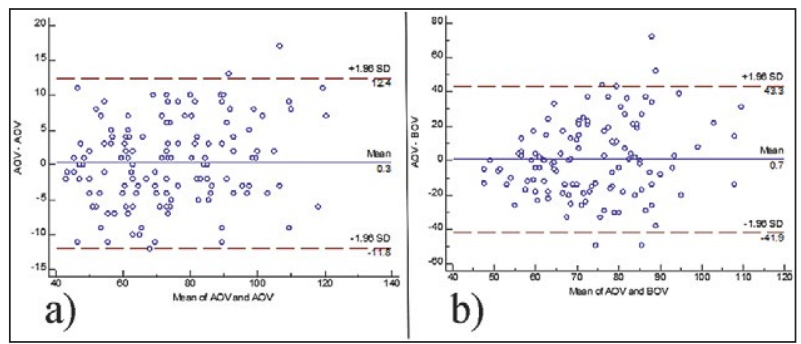

Fig 2. Bland-Altman plots indicate good reliability of intra-and inter- observer with Omniview technology. Top and bottom dashed lines show the $95 \%$ limits of agreement; a middle line shows mean difference: a) intra-observer reliability; b) interobserver reliability. Omniview technology acquisition time (AOV and $\mathrm{BOV}$ ) for both physicians (A and $\mathrm{B}$ ) and physician A used Omniview to reacquire images and record a new acquisition time (AOV'). 
inter-observer agreement, the Bland-Altman plots (fig $2 b$ ) indicate good agreement between physician A and B, the mean difference being $0.7 \mathrm{~s}$, LoA: -41.9 to $+43.3 \mathrm{~s}$.

\section{Image quality of $2 \mathrm{D}$ ultrasound imaging and Omniview technology}

Of the 189 fetuses, in 54 fetuses the corpus callosum images was successfully obtained using both 2D and Omniview technology. The scores of 2D ultrasound and Omniview images were $2.76 \pm 0.47$ and $2.80 \pm 0.41$, respectively. The Kappa score of 0.782 indicated a good agreement between the two imaging methods in the image quality (95\% CI: 0.586-0.977).

\section{Discussions}

Compared with traditional 2DUS, in 3DUS the sagittal, coronal and transverse sections of the fetal brain can be easily obtained allowing the reconstruction of the brain during examination or offline. The transabdominal $3 \mathrm{D}$ reconstruction of the fetal head can provide detailed evaluation of the fetal brain anatomy $[8,15]$. However, the corpus callosum and the cavum septi pellucidi could not be clearly distinguished in the sagittal section reconstructed by the biparietal horizontal cross-section [16] Plasencia et al [17] used 3D transabdominal ultrasound in 150 fetuses of 20-23 weeks of gestational age and found that the fetal corpus callosum could easily be obtained in the fetus facing the probe, exhibiting a hypoechoic band strip. It was also demonstrated that the display rate of the corpus callosum was also different after the corpus callosum was reconstructed through different angles and planes. Vinals et al [18] obtained a clear corpus callosum sonogram with the frontal suture as the translucent window and the use of tomography ultrasound imaging. The display rate and clarity of the corpus callosum were all higher compared with the other abdominal 3D methods.

We found that, the acquisition time of the corpus callosum was longer for the junior physician than for the senior physician only in 2DUS but not for Omniview technology. As long as the initial plane of the coronal plane of the fetus head is obtained, the corpus callosum can be observed through a simple operation. Hence, the image acquisition time for the corpus callosum was significantly shorter with the Omniview technology, compared with 2D ultrasound imaging for both operators. Furthermore, Omniview technology has good intra- and inter-observer reliability. Moreover, both junior and senior physicians can obtain satisfactory images of the corpus callosum in short time periods through a simple learning operation.

2D ultrasound imaging featured a lower success rate in obtaining an appropriate view of the corpus callosum comparing with Omniview, for both physicians. There are some explanations for the difficulties in obtaining a median sagittal plane: the fetus is often in cephalic presentation, the position of the fetal head is low and the manipulation of the probe is limited due to the influence of the pelvis. If the fetus is in breech presentation, the fetal head is tightly attached to the ribs and the manipulation of the probe can be limited also in this situation due to the influence of the rib. Only when the probe has enough space to be manipulated a proper fetal median sagittal plane can be obtained for a good corpus callosum image. Omniview technology is insensitive to the fetal position. However, the entire corpus callosum may be revealed using Omniview technology, if the coronary section could be obtained. Hence, Omniview technology may overcome the shortcomings of 2DUS imaging in visualizing the entire corpus callosum.

Omniview technology can obtain the imaging of the corpus callosum in a short period of time. As far as the quality of the image is concerned, there is no statistical difference in image quality, when compared with 2D ultrasound imaging. With the Omniview technology, the contour of the corpus callosum and the delineation from the cavum septi pellucidi was clear. Furthermore, the genu, body and splenium of the corpus callosum may be clearly distinguished, and the vermis of the cerebellum could be clearly observed.

One of the limitations of our study is concerned with the factors that can interfere with image quality (maternal abdominal wall thickness, gestational age, amniotic fluid abnormality, maternal respiration, fetal movement), factors that were not independently analyzed, Also, in this study, only the linear tracing method was used to obtain the midsagittal plane. The comparison between the results using this method and the use of curve, polyline or trace methods was not realized.

\section{Conclusion}

Compared with traditional 2DUS, the Omniview technology has some advantages in displaying the corpus callosum in the fetus during the second and third trimesters of pregnancy. It cannot only obtain corpus callosum image more effectively but also can achieve the quality standards of 2D images. Therefore, the Omniview technology can be used to rapidly and clearly image the corpus callosum.

Acknowledgment: This study was supported by the Key R \& D project of Shandong Province of China (2015GSF118081 and 2016GSF201141).

Conflict of interest: none 


\section{References}

1. Schoenemann PT, Sheehan MJ, Glotzer LD. Prefrontal white matter volume is disproportionately larger in humans than in other primates. Nat Neurosci 2005;8:242-252.

2. Luders E, Thompson PM, Toga AW. The Development of the Corpus Callosum in the Healthy Human Brain. J Neurosci 2010;30:10985-10990.

3. Paul LK, Brown WS, Adolphs R, et al. Agenesis of the corpus callosum: genetic, developmental and functional aspects of connectivity. Nat Rev Neurosci 2007;8:287-299.

4. Witelson SF. Hand and sex differences in the isthmus and genu of the human corpus callosum. A postmortem morphological study. Brain 1989;112:799-835.

5. Malinger G, Lev D, Lerman-Sagie T. Is fetal magnetic resonance imaging superior to neurosonography for detection of brain anomalies? Ultrasound Obstet Gynecol 2002;20:317-321.

6. D'Ettore A, Sole E, D'Armiento M, et al. Reliability of ultrasound examination confirmed at autopsy in fetuses suffering from Dandy-Walker Syndrome. Minerva Ginecol 2003;55:63-67.

7. Pilu G, Ghi T, Carletti A, Segata M, Perolo A, Rizzo N. Three-dimensional ultrasound examination of the fetal central nervous system[J].Ultrasound Obstet Gynecol; 2007;30:233-245.

8. Ghi T, Casadio P, Kuleva M, et al. Accuracy of three-dimensional ultrasound in diagnosis and classification of congenital uterine anomalies. Fertil Steril 2009;92:808-813.

9. Dunhua Z, Cai AL, Liu W, et al. Evaluation of the fetal corpus callosum with three-dimensional ultrasound multiplannar reconstruction. Chinese Journal of Medical Imaging Technology 2012;28:328-331.
10. Pilu G, Segata M, Ghi T, et al. Diagnosis of midline anomalies of the fetal brain with the three-dimensional median view. Ultrasound Obstet Gynecol 2006;27:522-529.

11. Viñals F, Muñoz M, Naveas R, Shalper J, Giuliano A. The fetal cerebellar vermis: anatomy and biometric assessment using volume contrast imaging in the C-plane (VCI-C). Ultrasound Obstet Gynecol 2005;26:622-627.

12. Zalel Y, Yagel S, Achiron R, Kivilevich Z, Gindes L. Threedimensional ultrasonography of the fetal vermis at 18 to 26 weeks' gestation: time of appearance of the primary fissure. J Ultrasound Med 2009;28:1-8.

13. Tonni G, Lituania M. OmniView algorithm: a novel 3-dimensional sonographic technique in the study of the fetal hard and soft palates. J Ultraound Med 2012;31:313-318.

14. [14] Rizzo G, Capponi A, Pietrolucci ME, et al. An algorithm based on OmniView technology to reconstruct sagittal and coronal planes of the fetal brain from volume datasets acquired by three-dimensional ultrasound. Ultrasound Obstet Gynecol 2011;38:158-164.

15. Correa FF, Lara C, Bellver J, Remohí J, Pellicer A, Serra V. Examination of the fetal brain by transabdominal three-dimensional ultrasound: potential for routine neurosonographic studies. Ultrasound Obstet Gynecol 2006;27:503-508.

16. Malinger G, Lerman-Sagie T, Vinals F. Three-dimensional reconstruction of the corpus callosum:fact or artifact. Ultrasound Obstet Gynecol 2006;28:742-743.

17. Plasencia W, Dagklis T, Borenstein M, Csapo B, Nicolaides KH. Assessment of the corpus callosum at 20-24 weeks' gestation by three-dimensional ultrasound examination.U1trasound Obstet Gynecol 2007;30:169-172.

18. Vinals F, Munoz M, Naveas R, Giuliano A. Transfrontal three-dimensional visualization of midline cerebral structures. Ultrasound Obstet Gynecol 2007;30:162-168. 